\title{
Role of Salivary Biomarkers in Systemic Diseases-A Systemic Review
}

\author{
Dr. Tarun Vyas ${ }^{1}$, Dr. Pradeep Vishnoi ${ }^{2}$, Dr. Kirti Choudhary ${ }^{3}$ \\ ${ }^{1}$ Asst. Professor, Department of Oral Medicine and Radiology R.R. Dental College \& Hospital, Umarda, \\ Udaipur Rajasthan, India. \\ E-mail: tarunvyas14@gmail.com \\ ${ }^{2}$ Asst. Professor, R.R. Dental College and Hospital, Udaipur Rajasthan, India. \\ ${ }^{3}$ Asst. Professor, RNT Govt. Medical College and Hospital, Udaipur Rajasthan, India.
}

\begin{abstract}
Salivary diagnosis is an increasingly important field in dentistry, physiology, internal medicine, endocrinology, pediatrics, immunology, clinical pathology, forensic medicine, psychology, and sports medicine. A growing number of drugs, hormones, and antibodies can be reliably monitored in saliva, which is an easily obtainable, non-invasive diagnostic medium It has become apparent that many systemic diseases affect salivary gland function and salivary composition. Studies of the effects of systemic diseases on salivary variables have been valuable in understanding the pathogenesis of the diseases, but their use as diagnostic markers has been limited. This article aims to review the point in the literature role of salivary biomarkers in systemic diseases.
\end{abstract}

Keywords: Saliva, Autoimmune Diseases, Malignancy, Hereditary, Forensic.

\section{Introduction}

Early detection of disease plays a crucial role in successful therapy. In most cases, the earlier the disease is diagnosed, the more likely it is to be successfully cured or well controlled. Managing a disease, especially in the early stage, may dramatically reduce the severity of its impact on the patient's life, or prevent and/or delay subsequent complications.

Most systemic diseases such as cancer, cardiovascular, metabolic, and neurological diseases are very challenging to diagnose without supplementary clinical evaluation. Even with a complete workup, the diagnosis usually remains uncertain due to complications of the disease. Currently, three major limitations have prevented people from recognizing the full potential of disease detection, and have seriously hampered the development of clinical diagnostics, namely:

1. Lack of definitive molecular biomarkers for specific diseases;

2. Lack of an easy and inexpensive sampling method with minimal discomfort; and

3. Lack of an accurate, easy-to-use, and portable platform to facilitate early disease detection. 
Since 2002, the National Institute of Dental and Craniofacial Research (NIDCR) created opportunities to overcome these limitations by exploring oral fluids as a diagnostic tool for assessment of health and disease status. Saliva, an oral fluid that contains an abundance of proteins and genetic molecules and is readily accessible via a totally noninvasive approach, has long been recognized as the potential solution to limitation number 2.

Through the visionary investment by the NIDCR, the discovery of salivary biomarkers and ongoing development of salivary diagnostics technologies now provide promising solutions for limitations 1 and 3.

With the additional advantages of an easy, safe, cost-effective, and non-invasive diagnostic approach, saliva shows high potential for monitoring general health and disease, with enormous translational values, and unparalleled opportunities for clinical applications.

There has been concern that although saliva contains diverse components with diagnostic properties, their low concentration compared with levels in the blood may prevent salivary diagnostics from being clinically practical; however, with the development of new and highly sensitive techniques (e.g., molecular diagnostics, nanotechnology), the low concentration of analytes in saliva is no longer a limitation. ${ }^{1}$

\section{Role of Salivary Biomarkers in Systemic Diseases}

Analysis of saliva done for the diagnosis of following -
A. Hereditary disease
Cystic fibrosis
Coelic disease
21-Hydroxylase deficiency
B. Autoimmune disease
C. Cardiovascular diseases
D. Malignancy
E. Infection

\section{Bacterial}

Helicobactor pylori infection

Pigeon breeder's disease (PBD)

Lyme disease

Viral

$$
\begin{aligned}
& \text { viral hepatitis } \\
& \text { mumps and rubella } \\
& \text { measles, } \\
& \text { Dengue } \\
& \text { HIV }
\end{aligned}
$$

\section{A. Hereditary Diseases}

1. Cystic Fibrosis: It is a genetically transmitted disease of children and young adults, which is considered a generalized exocrinopathy. It is the most common lethal autosomal-recessive disorder in Caucasians in North America, with an incidence of 1 in 2500 and a carrier frequency of 1 in 25-30 of the population. The gene defect causing this is present on chromosome 7 and codes for a transmembrane-regulating protein called the cystic fibrosis transmembrane conductance regulator (CFTR. Since a large number of identified 
mutations in the CF gene exist. The DNA analysis is not used for the diagnosis of the disease. The diagnosis is derived from the characteristic clinical signs and symptoms and analysis of elevated sweat chloride values. ${ }^{2}$

The abnormal secretions present in CF caused clinicians to explore the usefulness of saliva for the diagnosis of the disease. The submandibular saliva of CF patients was also found to contain more lipid than saliva of non-affected individuals and the levels of neutral lipds, phospholipids and glycolipids are elevated. These alterations in salivary lipids in CF patients may account, in part, for the altered physic-chemical properties of saliva in this disease ${ }^{3}$. Decreased protease activity in saliva from CF patients was observed relative to healthy controls, however, significant overlap between the protease activity values in the two groups was detected, which makes the diagnostic significance of these findings questionable ${ }^{4}$ Saliva from CF patients was found to contain an unusual form of epidermal growth factor (EGF). The EGF from these patients demonstrated poor biological activity compared with EGF from healthy controls. It was suggested that this EGF anomaly might contribute to the pathology of CF (Aubert et al, 1990). Further, abnormally elevated levels of prostaglandins $\mathrm{E}_{2}\left(\mathrm{PGE}_{2}\right)$ were detected in the saliva of $\mathrm{CF}$ patients as compared with that of healthy controls (Rigas et al, 1989). However, the diagnostic and clinical importance of the EGF anomaly and elevated salivary levels of $\mathrm{PGE}_{2}$ is difficult to interpret, since the role of $\mathrm{EGF}$ and $\mathrm{PGE}_{2}$ in the pathogenesis of CF is not defined.

Most of the studies concerning the diagnostic applications of saliva for CF are relatively old and saliva is not currently used for the diagnosis of this disorder. More important perhaps than the identification of diseased individuals is the detection of carriers (heterozygotes) for the disease, which are asymptomatic and cannot be detected by salivary or other biochemical diagnostic tests. Detection of carriers will help to reduce the incidence of CF. Screening for these carriers can be performed only at the DNA level. ${ }^{2}$

2. Coelic Disease: It is a congenital disorder of the small intestine that involves malabsorption of gluten. Gliadin is a major component of gluten. Serum IgA antigliadin antibodies5. No obvious explanation for the different between the two studies is apparent since both reports were similar in both methods of patient evaluation and salivary analysis. In a more recent study, salivary IgA-AGA produced a sensitivity of $60 \%$ and specificity of $93.3 \%$ in the detection of celiac disease. In comparison, serum IgA-AGA produced excellent sensitivity (100\%) but lower specificity (63.3\%). Because of the relative lower sensitivity, the authors did not recommend the use of salivary IgA-AGA for a screening of coeliac disease (Rujner et al, 1996). ${ }^{2}$

\section{21-Hydroxylase Deficiency:}

\section{B. Autoimmune Diseases- Sjogren's Syndrome}

Sjogren's syndrome (SS) is an autoimmune exocrinopathy of unknown etiology. The majority of patients are women and the estimated prevalence of the disease in the United States is more than 1 million. A reduction in lacrimal and salivary secretions is observed, associated with keratoconjunctivitis sicca and xerostomia. The presence of these two phenomena leads to a diagnosis of primary SS. In secondary SS, a well-defined connective tissue disease (most commonly rheumatoid arthritis or systemic lupus erythematosus) is present in addition to the xerostomia and/or the keratoconjunctivitis ${ }^{6}$ In addition to involvement of the salivary and lacrimal glands, SS may also affect the skin, lungs, kidneys, thyroid and nervous system ${ }^{7}$ ). The diagnostic criteria for SS are still uncertain and a single marker that is associated with all cases does not exist. The accepted procedure for the diagnosis of the salivary involvement of SS is a biopsy of the minor salivary glands of the lip. 
Serum chemistry can demonstrate polyclonal hypergammaglobulinemia and elevated levels of rheumatoid factor, antinuclear antibody, anti-SS-A and anti-SS-B antibody 8,9. The immunologic mechanisms involved in the pathogenesis of the disease appear also to involve B-cells (the majority of lymphomas associated with SS are of the B-cell type), salivary epithelial cells, an activated mononuclear cell infiltrate, cytokines and adhesion molecules (Fox and Speight, 1996).

Sialochemistry may also be used to assist in the diagnosis of SS. A consistent finding is increased concentrations of sodium and chloride. This increase is evident in both whole and gland-specific saliva ${ }^{10}$. In addition, elevated levels of IgA, IgG, lactoferrin and albumin and a decreased concentration of phosphate were reported in saliva of patients with SS (Ben-Aryeh et al 1981; Stuchell et al, 1984).

Analysis of unstimulated whole saliva was more sensitive than analysis of stimulated whole saliva for detection of these changes, since stimulation caused the elevated levels of sodium and IgA seen in SS patients to decline to the levels observed in healthy controls (Nahir et al, 1987). In contrast, normal concentrations of potassium and calcium are usually found in the saliva of SS patients. Although the amylase concentration in saliva is also normal, the production of amylase is reduced, but so is the amount of fluid. Therefore, measurement of amylase is not useful for the evaluation of salivary gland function in SS patients. ${ }^{11}$

SS is characterized by auto antibodies to the La \& Ro ribonucleoprotein antigens. These autoantibodies have been shown to target intracellular proteins which may be involved in the regulation of RNA polymerase function (Tan, 1989). Autoantibody, especially of the IgA class, can be synthesized in salivary glands and can be detected in the saliva of SS patients prior to detection in the serum ${ }^{12}$. In addition to IgA, saliva has also been reported to contain IgG autoantibody, while serum contained primarily IgG and IgM autoantibody. ${ }^{13}$

SS anti-La antibodies were primarily found in the saliva of patients whose resting and stimulated whole saliva flow rates were abnormally low. Furthermore, a strong correlation was observed between the presence of this autoantibody in serum and that in saliva. However, in some patients, the antibody was detected in whole saliva but not in serum, which suggested that the antibody is produced in the salivary glands ${ }^{14}$. The deposition of this antibody within salivary gland tissue may contribute to the pathogenesis of SS.

The diagnosis and early detection of SS present a serious challenge that has still not been met. Since no single salivary or serum constituent can accurately serve as a diagnostic marker for SS, the most important aspect of salivary diagnosis for this disease is evaluation of the reduced quantity of saliva. Cut -off values of $0.1 \mathrm{ml} / \mathrm{min}$ for resting whole saliva and $0.5 \mathrm{ml} / \mathrm{min}$ for stimulated saliva may be considered as indicative of salivary gland hypo-function ${ }^{15}$. Nevertheless, general agreement about these cut-off values does not exist.

Although variations in these cut-off values between clinicians may lead to differences in sensitivity and specificity in the diagnosis of SS, the quantitative evaluation of resting and stimulated saliva is a simple, noninvasive method of screening for patients who may have SS. Reduced salivary flow, although not pathognomonic for SS, is of clinical importance and can lead to a variety of oral signs and symptoms such as progressive dental caries, fungal infections, oral pain and dysphagia (Daniels and fox, 1992). Dentists are normally the first to encounter these patients. Affected individuals should be referred for a comprehensive evaluation of the cause for the reduced salivary flow. ${ }^{2}$ 


\section{Cardiovascular Disease}

Inflammation has been identified as the underlying cause of atherosclerosis, a condition that is associated with the deposition of lipids in the lining of arteries and progressively leads to acute myocardial infarction (AMI), also termed heart attack. A substantial number of patients with heart disease lack the established risk factors (increased lipids, hypertension, and family history). Unlike people with increased LDL cholesterol, people with increased concentrations of blood C-reactive protein (CRP), an acute-phase reactant produced by the liver during inflammatory processes, are unaware that they are susceptible to developing CVD and therefore may not seek medical advice to lower their risk. An increased CRP concentration warrants further investigation because it could be due to CVD. A recent randomized double-blinded trial JUPITER (Justification for the Use of statins in Prevention: An Intervention Trial Evaluating Rosuvastatin), which included more than 17000 "apparently healthy" women and men from 26 countries, revealed that in apparently healthy persons without hyperlipidemia but with increased CRP concentrations, prescription of rosuvastatin (a lipid-lowering drug) significantly reduced the incidence of major cardiovascular events.

Clinicians are currently considering whether to include CRP measurement as an additional risk factor in the Framingham cardiovascular risk factor calculator. Importantly, 2 recent studies by Christodoulides et al. and Dillon et al. have demonstrated that it is possible to detect CRP in saliva. The presence of CRP molecules in saliva provides an opportunity for the development of noninvasive assessments of CVD risk. However, salivary CRP reference ranges and their correlation with serum concentrations have not yet been investigated in detail. The low concentration of CRP in saliva dictates the need for analytically sensitive detection technologies.

Patients with acute chest pain suspected of having an AMI present an important diagnostic, economic, and operational challenge. Today's clinical work flow for an AMI patient includes an electrocardiogram at the initial screening, with a blood sample drawn and sent to a clinical laboratory for the analysis of cardiacspecific biomolecules.

AMI is defined by detection of the rise and/or fall of cardiac troponin (cTn), with at least 1 value above the 99th percentile of the upper reference limit, together with evidence of myocardial ischemia. Despite tremendous advancements, a substantial number of AMI cases are missed or diagnosed too late to offer effective therapies, accounting for high mortalities in Western societies. According to a recent report, in many emergency rooms examination for suspected AMI takes approximately $60 \mathrm{~min}$ for $25 \%$ of patients. A recent study by Floriano et al. documented the presence of cTnI in saliva, but with a poor diagnostic capability. This is the only reported study that investigated the diagnostic capability of saliva in AMI patients. ${ }^{16}$

\section{Malignancy}

Salivary analysis may aid in the early detection of certain malignant tumors. P53 is a tumor suppressor protein which is produced in cells exposed to various types of DNA-damaging stress. Inactivation of this suppressor through mutations and gene deletion is considered a frequent occurrence in the development of human cancer; ${ }^{17}$ It is clear that several risk factors are implicated in the development of oral cancer, of which the most common and established are tobacco smoking and alcohol. Hence, it is important for the public and the clinicians to be completely aware of the risk factors for oral cancer and it is prudent for dentists to look carefully for early signs of oral cancer. ${ }^{18}$ 
Defensins are peptides which possess antimicrobial and cytotoxic properties. They are found in the azurophil granules of polymorphonuclear leucocytes (Lichtestein, 1986; Lehrer et al, 1991). Elevated levels of salivary defensin-I were found to be indicative of the presence of oral SCC. Higher concentrations of salivary defensin-I were detected in patients with oral SCC in comparison with the defensin-I concentration in the saliva of patients with adenocarcinoma and in healthy controls. A high-positive correlation was observed between salivary defensin-I levels and serum levels of SCC-related antigen ${ }^{19}$.

A study by Navarro et al (1997) showed that EGF concentrations were higher in the saliva of women with primary breast cancer or a recurrence of breast cancer when compared with women without disease. Also, the profiling of salivary RNA transcript with microarray analysis seems to be able to distinguish several genes exhibiting significantly different expression levels in saliva comparing oral squamous cell carcinoma patients with controls ${ }^{20}$. It was also concluded that bidi smokers show higher cellular proliferation as compared to nonsmokers, which is similar to results obtained for cigarette smokers. However, it is not clear whether bidi smokers are at a greater risk of malignant transformation to OSCC than cigarette smokers. Further long-term studies which include large sample sizes comparing bidi and cigarette smokers need to be carried out to determine this. The lower plasma lipid status may be a useful bio-marker indicator for initial changes occurring in neoplastic cells..21,22

CA 125 is a tumor marker for epithelial ovarian cancer. Elevated salivary levels of CA 125 were detected in patients with epithelial ovarian cancer as compared to patients with benign pelvic masses and healthy controls. A positive correlation was found between salivary and serum levels of CA 125. A further analysis of this relationship revealed that saliva demonstrated a somewhat lower sensitivity than serum $(81.3 \%$ vs 93.8\% respectively); however, the specificity and positive predictive value were higher for saliva vs serum (88.0\% vs $59.8 \%$ and $54.2 \%$ vs $28.8 \%$, respectively; Chien and Schwartz, 1990). ${ }^{2}$

\section{E. Infectious Diseases}

\section{1). Bacterial Infections}

a. Helicobactor pylori infection is associated with peptic ulcer disease and chronic gastritis. Infection with this bacterium stimulates the production of specific IgG antibody. An ELISA test for the detection of IgG antibody in serum produced Pigeon breeder's disease (PBD) is an interstitial lung disease induced by exposure to antigens derived from pigeons. Measurement of salivary IgG against these antigens may assist in the evaluation of patients with this disease.

C. Lyme disease is caused by the spirochete Borrelia burgdorferi and is transmitted to humans by bloodfeeding ticks. The detection of anti-tick antibody in saliva has potential as a biologic marker of exposure to tick bites, which in turn may serve as a screening mechanism for individuals at risk for Lyme disease. ${ }^{23}$

Specific antibody to Taenia solium larvae in serum demonstrated greater sensitivity than antibody in saliva for identification of neurocysticercosis $(100 \%$ vs $70.4 \%$, respectively). However, considering the simple and non invasive nature of saliva sampling, it was suggested that saliva could be used in epidemiologic studies of this disease (Feldman et al., 1990). ${ }^{2}$

\section{Viral Disease}

The antibody response to infection is the basis for many diagnostic tests in virology. Saliva contains immunoglobulins that originate from two sources: the salivary glands and serum. The predominant 
immunoglobulin in saliva is secretory IgA ( $\operatorname{sg} A)$, which is derived from plasma cells in the salivary glands and constitutes the main specific immune defense mechanism in saliva.

Although the minor salivary glands play an important role in sIgA-mediated immunity of the oral cavity, cells in the parotid and submandibular glands are responsible for majority of the IgA found in saliva (Bienenstock et al, 1980). In contrast salivary IgM and IgG are primarily derived from serum via GCF and are present in lower concentrations in salivathan is IgA. Antibodies against viruses and viral components can be detected in saliva and can aid in the diagnosis of acute viral infections, congenital infections and reactivation of infection ${ }^{24}$ Saliva was found to be a useful alternative to serum for the diagnosis of viral hepatitis. Acute hepatitis A (HAV) and hepatitis B (HBV) were diagnosed based on the presence of IgM antibodies in saliva. The ratio of IgM to IgG anti-HAV antibody correlated with the time interval from onset of infection (Parry et al, 1989). Further, salivary antibody levels were used for the detection of infected individuals in a school outbreak of HAV 25.

Saliva has also been utilized to detect very low levels of antibodies to HAV, which, for example, are associated with vaccine-induced immunity. Comparison of serum and saliva levels of antibody to HAV revealed excellent agreement (Sensitivity $=98.7 \%$ and specificity $=99.6 \%$, Ochnio et al, 1997). Similarly, analysis of saliva provided a highly sensitive and specific method for the diagnosis of viral hepatitis B and C (El-Medany et al, 1999). Sensitivity and specificity of $100 \%$ for the detection of antibodies for both diseases in oral fluid in comparison with serum antibodies were reported 26.

Saliva has also been used for screening for hepatitis B surface antigen (HbsAg) in epidemiologicall studies. Comparing the detection of $\mathrm{HbsAg}$ in saliva with that in serum by means of a commercially available serological kit yielded a sensitivity of $92 \%$ and specificity of $86.7 \%$ (Chaita et al, 1995).

Saliva may also be used for determining immunization and detecting infection with measles, mumps and rubella (Friedman, 1982; Perry et al, 1993; Brown et al, 1994) 27 . The detection of antibodies in oral fluid samples produced sensitivity and specificity of $97 \%$ and $100 \%$ for measles, $94 \%$ and $94 \%$ for mumps and $98 \%$ and $98 \%$ for rubella, respectively, in comparison with detection of serum antibodies for these viruses (Thieme at al, 1994).

Dengue is a mosquito-transmitted viral disease. Primary infection of the virus may lead to a self-limiting febrile disease and secondary infection may cause serious complications like dengue hemorrhagic fever or dengue shock syndrome (Burke et al, 1988). Salivary levels of anti-dengue IgM and IgG demonstrated sensitivity of $92 \%$ and specificity of $100 \%$ in the diagnosis of primary and secondary infection and salivary levels of IgG proved useful in differentiating between primary and secondary infection (Cuzzubbo et al., 1998). Saliva was also found to be a reliable alternative to serum for identification of the antibody to parvovirus B 19. Sensitivity of $100 \%$ and specificity of $95 \%$ were observed for the detection of infected individuals at a primary school (Rica and Cohen, 1996). ${ }^{2}$

HIV: Studies have demonstrated that the diagnosis of infection with the human immunodefiency virus (HIV) based on specific antibody in saliva is equivalent to serum in accuracy and therefore applicable for both clinical use and epidemiological surveillance (Malamud, 1992). Antibody to HIV in whole saliva of infected individuals, which was detected by ELISA and Western Blot Assay, correlated with serum antibody levels ${ }^{28}$ As compared with serum, the sensitivity and specificity of antibody to HIV in saliva for detection of infection are between $95 \%$ and $100 \%$ ). Salivary IgA levels to HIV decline as infected patients become symptomatic. It 
was suggested that detection of IgA antibody to HIV in saliva, may, therefore, be a prognostic indicator for the progression of HIV infection (Matsuda et al., 1993).

Analysis of antibody in saliva as a diagnostic test foe HIV (or other infections) offers several distinctive advantages when compared with serum. Saliva can be collected non-invasively, which eliminates the risk of infection for the health care worker who collects the blood sample. Furthermore, viral transmission via saliva is unlikely, since infectious virus is rarely isolated from saliva (Ho et al., 1985). Saliva collection also simplifies the diagnostic process in special populations in whom blood drawing is difficult, i.e., individuals with compromised venous access (e.g., injecting drug users), patients with hemophilia and children (Archibald et al., 1993).

Several salivary and oral fluid tests have been developed for HIV diagnosis. Orasure is a testing system that is commercially available in the United Staes and can be used for the diagnosis of HIV. The test relies on the collection of an oral mucosal transudate (and therefore IgG antibody)., 1993; Gaudette et al., 1994). Different oral pathologic lesions, which are relatively common in HIV-infected individuals, do not appear to influence the results ${ }^{29}$. In conclusion, collection and analysis of saliva offer a simple, safe, well-tolerated and accurate method for the diagnosis of HIV infection. ${ }^{2}$

\section{Role of saliva in Monitoring of levels of drugs}

Therapeutic drugs

Psychiatric medications

Anti-epileptic drugs.

Anti-cancer drugs

Drug abuse/ Recreational drugs.

\section{Drug Monitoring}

Similar to other body fluids (i.e. serum, urine and sweat), saliva has been proposed for the monitoring of systemic levels of drugs ${ }^{30}$ Therefore, drugs which are not ionizable or are not ionized within the ph range of saliva, are the most suited to salivary drug monitoring. Due to their size, serum-binding proteins do not cross the membrane. Therefore, only the unbound fraction of the drug in serum is available for diffusion into saliva (Haeckel, 1993). The unbound fraction of a drug is usually the pharmacologically active fraction. This may represent an advantage of drug monitoring in saliva in comparison with drug monitoring in serum, where both bound and unbound fractions of a drug can be detected.Other parameters which may influence the availability of drugs in saliva are the mechanism of drug transfer into saliva (since some drugs reach saliva in ways other than passive diffusion), salivary flow rate (increased flow rate affects salivary ph by increasing bicarbonate secretion) and drug stability in saliva. ${ }^{2}$

\section{Drug Monitoring in Saliva}

Therapeutic drugs: Antipyrine, Caffeine, Diazepam, Digoxin, Phenytoin, Quinine, Sulfonilamide, Theophylline, Tolbutamide, Parecetamol, Cyclosporine, Carbamazepine.

Drug abuse/ Recreational drugs: Amphetamines, Barbiturates, Cocaine, Nicotine, Opiods, Ethanol.

Saliva may be used for monitoring patient compliance with psychiatric medications. A significant correlation exists between the salivary and serum lithium levels in patients receiving lithium therapy. ${ }^{31}$ 
Saliva is also useful for the monitoring of anti-epileptic drugs. Salivary carbamazepine levels were found to be $38 \%$ of serum carbamazepine levels and a positive correlation between salivary and serum carbamazepine levels was observed. Stimulation of salivary flow and storage of saliva for several days did not affect this correlation (Rosenthal et al, 1995). In another study, salivary levels of Phenobarbital and phenytoin demonstrated excellent correlations with serum level of these medications. A lower correlation was found between salivary and total serum levels of cyclosporine. Cyclosporine is a neutral lipophilic molecule that enters saliva mostly be passive diffusion and salivary levels of this drug reflect the serum levels of free cyclosporine. Therefore, salivary cyclosporine levels may correlate better with serum levels of free, rather than total, cyclosporine (Coates et al., 1988). Similarly, salivary theophylline concentration demonstrated a better correlation with serum concentration of free theophylline than with serum concentration of total theophylline. ${ }^{32}$

Saliva may also be used for monitoring levels of anti-cancer drugs. Saliva was found to be a reliable alternative to serum for the monitoring of irinotecan levels. Salivary analysis may be used to evaluate the cisplastin concentration in serum; however, a defined correlation between salivary and serum levels was not reported ${ }^{33}$. Conversely, serum carboplatin concentration demonstrated considerable variations and was found to be unreliable in measurements of serum carboplatin (Of particular interest is the use of saliva for the evaluation of illicit drug use. Following drug use, the appearance of the drug in saliva follows a time course that is similar to that of serum. In contrast, drugs appear at a later time point $n$ urine. Nevertheless, as opposed to what is needed for the monitoring of therapeutic drugs, the presence of illicit drugs and not their concentration, is usually sufficient for forensic purposes. One important exception is ethanol. Ethanol is not ionized in serum, is not protein-bound and due to its low molecular weight and lipid solubility, diffuses rapidly into saliva. A significant correlation between salivary and serum alcohol levels was reported (Penttila et al., 1990.Saliva can also be used to detect recent marijuana use by means of radioimmunoassay (Gross et al., 1985). Saliva can be used to monitor tobacco smoking and exposure to tobacco smoke. The major nicotine metabolite cotinine was investigated as an indicator of exposure to tobacco smoking. Cotinine is tobacco-specific and has a relatively long half life compared with nicotine.Salivary thiocyanate was also found to be an indicator of cigarette smoking (Luepkar et al., 1981); however, cotinine levels are considered the most reliable marker (Di Giust0 and Eckhard, 1986). ${ }^{2}$

\title{
Monitoring of Levels of Hormones
}

\author{
Aldosterone \\ Prednisone \\ Prednisolone \\ Salivary testosterone \\ Estradiol \\ Progesterone \\ Insulin
}

\section{Monitoring of Hormone levels}

Saliva can be analyzed as part of the evaluation of endocrine function. The factors that affect drug availability in saliva are generally true also for salivary hormones. The majority of hormones enter saliva by passive diffusion across the acinar cells.. The molecular weight cut-off for ultrafiltration is 100-200. This relatively small molecular size prevents many hormones from entering saliva from serum by means of ultrafiltration. Conversely, due to their size, protein hormones due to enter saliva through passive diffusion, but primarily through contamination from serum as a result of outflow of GCF or from oral wounds. Furthermore, some 
steroid hormones can be metabolized in the transcellular diffusion, which can affect availability of these hormones in saliva. 34

Due to their lipid solubility, steroid hormones can be detected in saliva. Salivary cortisol levels demonstrate excellent correlation with free serum cortisol levels(. This high correlation is not affected by changes in concentrations of serum binding proteins. However, the actual salivary cortisol levels are lower than the serum-free cortisol levels, possibly due to enzymatic degradation in the salivary epithelial cells during transcellular diffusion ${ }^{34}$ Salivary cortisol levels were found to be useful in identifying patients with Cushing's syndrome and Addison's disease, and also for monitoring the hormone response to physical exercise ${ }^{35}$ and the effect of acceleration stress. Thus, cortisone levels in saliva are higher than in serum and do not bear any diagnostic significance (Vining and McGinley, 1986). Other corticosteroids like prednisone and prednisolone also do not show a consistent correlation between serum and salivary levels, possibly due to the effect of the same enzyme.

Salivary aldosterone levels demonstrated a high correlation with serum aldosterone levels and increased aldosterone levels were foundin both the serum and saliva of patients with primary aldosteronism (Conn's syndrome;). A similar high correlation between salivary and serum aldosterone levels was observed with the use of a solid-phase enzyme immunoassay ${ }^{36}$ These findings were supported by an additional study and salivary aldosterone levels were found to be approximately one-third of serum level 0 . Testosterone and dehydroepiandrosterone have also been identified in saliva. Salivary concentrations were found to be 1.5$7.5 \%$ of the serum concentrations of these hormones. Similarly, salivary testosterone levels were detected in an additional study which proposed the use of salivary testosterone levels for the assessment of testicular function By a direct radioimmunoassay technique, a high correlation between salivary and serum-free testosterone concentration and salivary and serum total testosterone concentration was reported. A significant correlation between the concentration of unbound salivary and serum testosterone was observed when hormone levels in normal and hyperandrogenic women were evaluated. Monitoring salivary testosterone levels may also be useful in behavioral studies of ${ }^{37} \mathrm{~A}$ aggregation, depression, abuse and violent and antisocial behavior (high correlation between the salivary concentration of androstenedione and dihydrotestosterone and the unbound serum concentration of these hormones has also been reported.

Salivary monitoring of hormone levels has many advantages over the more conventional serum analysis. In addition to the other advantages of salivary diagnosis, hormone evaluation often necessitates multiple sample collection in a relatively short time interval, which makes the non-invasivecollection of saliva ideal for this purpose. 38

The stability of hormones in saliva is important as well for accurate evaluation. Hormones in saliva can be degraded from oral microorganisms and enzymes derived from leukocytes that enter the oral cavity from the gingival sulcus. In addition, molecules that reach saliva by passive diffusion across cells, like unconjugated steroids, may be subjected to enzymatic degradation within the salivary glands, prior to entering saliva ${ }^{34}$. These factors have to be considered when saliva is evaluated as an alternative for the evaluation of serum hormone levels. ${ }^{2}$

\section{Forensic Evidence}

Saliva may be found on victims of several violent crimes. Saliva can potentially be recovered from bite marks, cigarette butts, postage stamps, envelopes and other objects. Stains of dried saliva are invisible, making its recognition and collection difficult. However, the presence of saliva can be confirmed by 
amylase assay. During the biting process, saliva is deposited on the skin or object surface in enough amount to allow typing of the deoxyribonucleic acid (DNA). Dental and craniofacial radiograph are an important assessment tool in race, gender and stature due diversity in human physical constitutional makeup. ${ }^{39}$

Polymerase chain reaction (PCR) allows replication of thousands of copies of a specific DNA sequence in vitro, enabling the study of small amounts of DNA. ${ }^{40}$

\section{Conclusion}

Definition of specific disorders that can be identified or monitored by the analysis of saliva offers the possibility of improved patient management. Consequently, we are likely to see the increased utilization of saliva as a diagnostic fluid. As a result, dentists will have greater involvement in the identification and monitoring of certain non-oral disorders The potential advantages of salivary analysis for the diagnosis of systemic disease suggest that further studies are warranted.

\section{References}

1. Lee YH, Wong DT. Saliva: An emerging biofluid for early detection of diseases. Am J of Den. 2009; 22(4): 241-248.

2. Kaufman E, Lamster IB. The diagnostic applications of saliva-a review. Crit Rev Oral Biol Med. 2002; 13(2):197-212.

3. Slomiany BL, Aono M, Murty VL, Slomiany A, Levine MJ, Tabak LA (1982). Lipid composition of submandibular sali- va from normal and cystic fibrosis individuals. J Dent Res 61:1163-1166.

4. Kittang E, Odegaard OR, Michalsen H (1986). Cystic fibrosis: pro- tease activity in saliva evaluated with chromogenic substrates. Eur J Respir Dis 68:263-266.

5. Patinen P, Bjorksten F, Malmstrom M, Savilahti E, Reunala T (1995). Salivary and serum IgA antigliadin antibodies in der- matitis herpetiformis. Eur J Oral Sci 103:280-284.

6. Schiødt M, Thorn J (1989). Criteria for the salivary component of Sjögren's syndrome. A review. Clin Exp Rheumatol 7:119-122.

7. Talal N (1992). Sjögren's syndrome: historical overview and clini- cal spectrum of disease. Rheum Dis Clin North Am 18:507-515.

8. Atkinson JC, Travis WD, Pillemer SR, Bermudez D, Wolff A, Fox PC (1990). Major salivary gland function in primary Sjögren's syndrome and its relationship to clinical features. J Rheumatol 17:318-322.

9. Fox RI, Kang HI (1992). Pathogenesis of Sjögren's syndrome.Rheum Dis Clin North Am 18:517-538.

10. Tishler M, Yaron I, Shirazi I, Yaron M (1997). Saliva: an additional diagnostic tool in Sjögren's syndrome. Semin Arthritis Rheum 27:173-179.

11. Mandel ID (1980). Sialochemistry in diseases and clinical situa- tions affecting salivary glands. Crit Rev Clin Lab Sci 12:321-366.

12. Horsfall AC, Rose LM, Maini RN (1989). Autoantibody synthesis in salivary glands of Sjögren's syndrome patients. J Autoimmun 2:559-568. 
13. Ben-Chetrit E, Fischel R, Rubinow A (1993). Anti-SSA/Ro and anti-SSB/La antibodies in serum and saliva of patients with Sjögren's syndrome. Clin Rheumatol 12:471474.

14. Sreebny L, Zhu WX (1996b). Whole saliva and the diagnosis of Sjögren's syndrome: an evaluation of patients who complain of dry mouth and dry eyes. Part 2: Immunologic findings. Gerodontology 13:44-48.

15. Sreebny L, Zhu WX (1996a). Whole saliva and the diagnosis of Sjögren's syndrome: an evaluation of patients who complain of dry mouth and dry eyes. Part 1: Screening test. Gerodontology 13:35-43.

16. Pfaffe T, Cooper-White J, Beyerlein P, Kostner K, and Punyadeera C. Diagnostic Potential of Saliva:Current State and Future Applications. Clinic Chemi. 2011; 57(5): 675-687.

17. Tarapore P, Fukasawa K (2000). P53 mutation and mitotic infideli- ty. Cancer Invest 18:148-155.

18. Vyas, T., Kuthiala, P., \& Vishnoi, P. (2019). Oral Cancer: Etiology and Its Diagnostic Aids. International Journal of Drug Research And Dental Science, 1(2), 13-18.

19. Mizukawa N, Sugiyama K, Fukunaga J, Ueno T, Mishima K, Takagi S, et al. (1998). Defensin-1, a peptide detected in the saliva of oral squamous cell carcinoma patients. Anticancer Res 18:4645-4649.

20. Chiappin S, Antonelli G, Gatti R, De Palo EF. Saliva specimen: A new laboratory tool for diagnostic and basic investigation. Clinica Chimica Acta. 2007; 383: 30-40.

21. Vyas, T., Verma, P., Abidullah, M., Kushwaha, S. S., Sahoo, P. K., Priyadarshini, S. R., ... \& Rana, V. (2018). Quantitative analysis of AgNOR counts and pap stain in exfoliative cytology specimens of oral mucosa in bidi smokers and nonsmokers. Annals of African medicine, 17(4), 210-214.

22. Vyas T, Bhargava R and Sharma A. Comparative Study of Serum Lipid Profile Parameters for Oral Cancer and Non Oral Cancer Patients. Int J Com Health and Med Res 2016;2(2):49-55.

23. Schwartz BS, Ford DP, Childs JE, Rothman N, Thomas RJ (1991). Anti-tick saliva antibody: a biologic marker of tick exposure that is a risk factor for Lyme disease seropositivity. Am J Epidemiol 134:86-95.

24. Mortimer PP, Parry JV (1988). The use of saliva for viral diagnosis and screening. Epidemiol Infect 101:197-201.

25. Stuart JM, Majeed FA, Cartwright KA, Room R, Parry JV, Perry KR, et al. (1992). Salivary antibody testing in a school outbreak of hepatitis A. Epidemiol Infect 109:161-166.

26. Thieme T, Yoshihara P, Piacentini S, Beller M (1992). Clinical evaluation of oral fluid samples for diagnosis of viral hepatitis. J Clin Microbiol 30:1076-1079.

27. Kinney JS, Ramseher CA. Oral fluid-based biomarkers of alveolar bone loss in periodontitis. Ann. N.Y. Acad. Sci. 2007; 1098: 230-251.

28. Frerichs RR, Silarug N, Eskes N, Pagcharoenpol P, Rodklai A, Thangsupachai S, et al. (1994). Saliva-based HIV-antibody test- ing in Thailand. AIDS 8:885-894.

29. Gallo D, George JR, Fitchen JH, Goldstein AS, Hindahl MS (1997). Evaluation of a 
system using oral mucosal transudate for HIV- 1 antibody screening and confirmatory testing. J Am Med Assoc 277:254-258.

30. Drobitch RK, Svensson CK (1992). Therapeutic drug monitoring in saliva. An update. Clin Pharmacokinet 23:365-379.

31. Groschl M. The physiological role of hormones in saliva. BioEssays. 2009; 31: 843-852.

32. Kirk JK, Dupuis RE, Miles MV, Gaddy GD, Miranda-Massari JR, Williams DM (1994). Salivary theophylline monitoring: reassessment and clinical considerations. Ther Drug Monit 16:58-66.

33. Holding JD, Lindup WE, Roberts NB, Salvatori P, Stell PM (1999). Measurement of platinum in saliva of patients treated with cis- platin. Ann Clin Biochem 36:655-659.

34. Quissell DO (1993). Steroid hormone analysis in human saliva.Ann NY Acad Sci 694:143-145.

35. Lac G, Pantelidis D, Robert A (1997). Salivary cortisol response to a $30 \mathrm{mn}$ submaximal test adjusted to a constant heart rate. J Sports Med Phys Fitness 37:5660.

36. Hubl W, Taubert H, Freymann E, Hofmann F, Meissner D, Garten CD, et al. (1983). A simple solid phase enzyme immunoassay for aldosterone in plasma and saliva. Exp Clin Endocrinol 82:188-193.

37. Dabbs JM Jr, Campbell BC, Gladue BA, Midgley AR, Navarro MA, Read GF, et al. (1995). Reliability of salivary testos- terone measurements: a multicenter evaluation. Clin Chem 41:1581-1584.

38. Ellison PT (1993). Measurements of salivary progesterone. Ann NY Acad Sci 694:161-176.

39. Vyas T. Radiographic determination: An upcoming aid in forensic radiology. J Int Clin Dent Res Organ 2019;11:71-5.

40. Mittal S, Bansal V, Garg S, AtrejaG, Bansal S. The diagnostic role of Saliva - A Review. J Clin Exp Dent. 2011; 3(4): e314-e320.

How to cite this Article: Dr. Tarun Vyas ${ }^{1 *}$, Dr. Pradeep Vishnoi², Dr. Kirti Choudhary3; Role of Salivary Biomarkers in Systemic Diseases-A Systemic Review

Crossref doi: https://doi.org/10.36437/ijdrd.2019.1.3.I

Int. J. Drug Res. Dental Sci., 2019; 1(3):1-13.

Source of Support: Nil, Conflict of Interest: None declared. 